\title{
The Influence of Pre- and Postoperative Fear Avoidance Beliefs on Postoperative Pain and Disability in Patients With Lumbar Spinal Stenosis
}

\author{
Analysis of the Lumbar Spinal Outcome Study (LSOS) Data
}

Jakob M. Burgstaller, MD, DMD, ${ }^{*, \dagger}$ Maria M. Wertli, MD, PhD, ${ }^{*, \ddagger}$ Johann Steurer, MD, ${ }^{*}$ Alfons G.H. Kessels, MD, MSc, ${ }^{\dagger}$ Ulrike Held, PhD, ${ }^{*}$ and Hans-Fritz Gramke, MD, $\mathrm{PhD}^{\dagger}$, on behalf of the LSOS Study Group

Study Design. Prospective multicenter cohort study.

Objective. To evaluate the effect of pre- and postoperatively assessed fear avoidance beliefs (FAB) on pain and disability in patients with degenerative lumbar spinal stenosis (LSS) after decompression surgery.

Summary of Background Data. To the present, the influence of pre- and postoperative $\mathrm{FAB}$ on the prognosis after surgery for LLS is still unclear.

Methods. Patients of the Swiss Lumbar Stenosis Outcome Study (LSOS) with confirmed LSS undergoing first-time decompression without fusion were enrolled in this study. The main outcome of this study was minimal clinically important difference (MCID) in spinal stenosis measure symptoms (pain) and function (disability) after 12 months. To analyze the influence of pre- and postoperatively assessed FAB on pain and disability we built simple and multiple logistic regression models.

Results. In this analysis of 234 patients undergoing decompression surgery for symptomatic degenerative LSS we found baseline $\mathrm{FAB}$ measured by the FAB physical activity subscale (FABQP) not to be associated with pain (OR 0.95; 95\% Cl: $0.55-1.67$ ) and disability (OR 1.11; 95\% Cl: 0.64-1.92) at 12 months' $^{\prime}$

From the *Horten Centre for Patient Oriented Research and Knowledge Transfer, University of Zurich, Zurich, Switzerland; 'Department of Anesthesiology and Pain Therapy, University Hospital Maastricht, Maastricht, The Netherlands; and ${ }^{\ddagger}$ Department of General Internal Medicine, Bern University Hospital, Bern University, Bern, Switzerland.

Acknowledgment date: April 22, 2016. First revision date: June 24, 2016. Acceptance date: July 19, 2016.

The manuscript submitted does not contain information about medical device(s)/drug(s).

Baugarten Foundation, Helmut Horten Foundation, Pfizer-Foundation for geriatrics \& research in geriatrics the Symphasis Charitable Foundation, and the OPO Foundation funds were received in support of this work.

Relevant financial activities outside the submitted work: grants.

Address correspondence and reprint requests to Jakob M. Burgstaller, MD, DMD, Horten Centre for Patient Oriented Research and Knowledge Transfer, University of Zurich, Pestalozzistr. 24, 8091 Zurich, Switzerland;

E-mail: jakob.burgstaller@usz.ch

DOI: 10.1097/BRS.0000000000001845 follow-up. In the final multiple logistic regression models patients with high FABQ-P at 6 months (OR 0.46; 95\% Cl: 0.24-0.91) and high persistent FABQ-P at baseline and 6 months (OR 0.34, 95\% Cl: 0.16-0.73) were less likely to report a MCID for spinal stenosis measure symptoms at 12 months. Our analysis found a similar trend for disability; however, the results were not statistically significant.

Conclusion. In elderly patients undergoing decompression surgery for symptomatic degenerative LSS preoperative fear avoidance beliefs were not a prognostic indicator for the outcome. Patients with $\mathrm{FAB}$ at 6 months and persistent FAB were less likely to experience clinically relevant improvement in pain at 12 months. Studies should address the importance of persistent postoperative FAB.

Key words: decompression, fear, fear avoidance, laminotomy, lumbar spinal stenosis, lumbar spine, multicenter, outcome, spinal canal stenosis, surgery.

Level of Evidence: 3

Spine 2017;42:E425-E432

ow back pain (LBP) with its global prevalence of 9.4\% constitutes a high health and economic burden on individuals. ${ }^{1}$ Lumbar spinal stenosis (LSS) is an important subgroup of LBP patients. In the United States, LSS is the most frequent indication for spine surgery in patients older than 65 years. ${ }^{2}$ However, there is a wide variation in rates of improvement in patients after surgery. ${ }^{3,4}$ Various factors may influence chronic postsurgical complaints being a major concern because it can affect patient recovery and quality of life after surgery. ${ }^{5,6}$ Preoperatively assessed psychological factors including fear and pain catastrophizing were associated with more postoperative pain. ${ }^{7}$ On the other hand, high postoperative fear was also associated with poorer outcomes as postoperative pain intensity and disability. ${ }^{8}$ How fear and catastrophizing influence the postoperative course in patients with LSS has not yet fully understood.

www.spinejournal.com

E425 
The fear-avoidance model is a widely used theoretical model to explain psychological factors in the experience of pain and the development of chronic pain and disability. ${ }^{9}$ Negative beliefs about pain and/or negative illness information may lead to an exaggerated negative mental response where the worst possible outcome is imagined (pain catastrophizing). This catastrophizing response to a painful experience leads to pain-related fear and avoidance behaviors. Avoidance behavior results in disuse, disability, and depression, and maintains the original negative appraisal in a deleterious cycle. ${ }^{9}$ On the other hand, it is assumed that patients without catastrophizing thoughts and fear avoidance beliefs are more likely to confront pain and are more active in the coping process. ${ }^{9}$ While in nonspecific low back pain the importance of fear avoidance beliefs and catastrophizing thoughts on the prognosis and treatment efficacy has been shown, ${ }^{10-13}$ their influence in patients with LSS has not been investigated. How surgery influences postoperative fear avoidance beliefs is not well understood. One study showed a postoperative reduction in fear avoidance beliefs in patients undergoing surgery for degenerative spinal conditions. ${ }^{8}$ To date, no study investigated the influence of pre- and postoperative fear avoidance beliefs on the prognosis after surgery for LLS.

The aim of this study is to evaluate the effect of high preand postoperative fear avoidance beliefs on pain and disability in patients with degenerative lumbar spinal stenosis after decompression surgery.

\section{MATERIALS AND METHODS}

\section{Patient Selection}

We used data from the Lumbar Stenosis Outcome Study (LSOS), ${ }^{14}$ a multicenter prospective cohort study, to explore this issue. Patients with a history of neurogenic claudication were recruited from outpatient clinics at all participating centers. Eligible patients had no evidence of stenosis caused by tumor, fracture, infection, or significant deformity $\left(>15^{\circ}\right.$ lumbar scoliosis), and were aged 50 years or more. Magnetic resonance imaging verified lumbar spinal canal stenosis. None of the patients had prior lumbar spine surgery. Furthermore, patients had no clinical peripheral artery occlusive disease (confirmed by a vascular specialist in patients without palpable pulses in the lower limb).

\section{Surgical Procedure}

Surgery consisted of a standard open or microscopic posterior lumbar decompression at the affected level or levels without instrumentation.

\section{Questionnaires}

\section{Fear Avoidance Beliefs Questionnaire (FABQ)}

The FABQ ${ }^{15}$ is a 16 -item questionnaire. High values indicate increased levels of fear avoidance beliefs. Two subscales exist: a seven-item work subscale (FABQ-W; range $0-42$ ) and a four-item physical activity subscale (FABQ-P; range $0-24)$. The FABQ and the two subscales have been shown to be reliable and valid for the measurement of fear avoidance beliefs. The Cronbach $\alpha$ value for the FABQ-P was 0.75 (test-retest reliability, $r=0.64$ ) and for the FABQW0.82 (test-retest $r=0.80) .{ }^{16}$ In this study, we did not use the FABQ-W because most patients were retired.

\section{Spinal Stenosis Measure (SSM)}

The SSM, an instrument specifically developed for spinal stenosis patients by Stucki et al, ${ }^{17}$ targets to measure severity of symptoms and quantifies disability of the lumbar spinal stenosis population. This instrument is recommended by the North American Spine Society (NASS) and used in different studies on lumbar spinal stenosis. ${ }^{18-21}$ It consists of three different subscales; the symptom severity subscale (score range $1-5$, best-worst), the physical function subscale (1$4)$, and the satisfaction subscale (1-4).

\section{$E Q-5 D-3 L$}

The EQ-5D-3L is an assessment tool to quantify healthrelated quality of life. It quantifies general nondisease specific health-related quality of life, including physical, mental, and social dimensions. ${ }^{22}$ It can be calculated as a sum score (score range $0-100$, worst-best). ${ }^{22}$ The second part of the questionnaire estimates patient's actual health status (score range $0-100$, worst-best).

\section{Hospital Anxiety and Depression Scale (HADS)}

The HADS was originally developed to measure anxiety (score range $0-21$, best-worst) and depression $(0-21)$ in a hospital setting, ${ }^{23}$ however, it is nowadays common to use it in all settings. ${ }^{24}$

\section{Data Collection and Follow-up}

Parts of the basic data sheet were interview-administered and recorded by a study coordinator. All other questionnaires were self-administered and filled in by the patients themselves. All data were collected at baseline, 6 and 12 months.

\section{Prognostic Indicator}

We defined FABQ-P to qualify as a prognostic indicator if the score of FABQ-P influenced the course of the disease after adjustment for potential confounders.

\section{Cutoff Value for High FABQ-P}

We used a cutoff value for high FABQ-P of $>16$ points based on the 75 th percentile at 6 months according to Grotle et al. ${ }^{25}$

\section{Outcomes}

The main outcome of this study was clinically meaningful improvement in SSM symptoms and function, which is denoted as minimal clinically important difference (MCID), after 12 months.

The MCID is defined as "the smallest difference in a score that is considered to be worthwhile or important." 26 Thus, 
the MCID is a threshold for a relevant change in an outcome measure. Patients who reached or even exceed this threshold consider this change as meaningful and worthwhile. According to Stucki et al, ${ }^{17}$ MCID for SSM is reached when "Symptom Severity scale" improves at least 0.48 points and "Physical Function scale" at least 0.52 points at the 6- or 12-month follow-up.

\section{Statistical Analyses}

Analysis of data consisted of descriptive statistics of patient demographics and outcomes. Continuous and ordinal scaled variables were shown as median and interquartile ranges, and categorical variables were shown as numbers and percentages of total.

To analyze the influence of pre- and postoperatively assessed fear avoidance beliefs (FAB) on pain and disability we built different models for MCID in SSM symptoms and SSM function, respectively. First, continuous FABQ-P at baseline and continuous FABQ-P at 6 months, respectively, were the potential prognostic indicators in the models Ia and Ib. Dichotomous FABQ-P $>16$ at baseline (high FABQ-P-0) and FABQ-P $>16$ at 6 months (high FABQ-P-6), respectively, were entered in the models IIa and IIb. Persistent high FABQ-P $>16$ at baseline and at 6 months (high FABQ-P-persistent) entered in model III. In the models Ia and Ib, we first fitted a nonlinear effect within the generalized additive model framework in order to verify or falsify the linearity assumption of the continuous prognostic indicator.

Potential confounders were selected a priori in an interdisciplinary consensus (JB, MW, JS, AK, UH, HG) and based on the current literature. ${ }^{27,28}$ Demographic variables were age and sex (entered as continuous and categorical variable in the model, respectively), socioeconomic variables were education (low education defined as compulsory education), and civil status (single, divorced, and widowed as risk factor), general health (presence of comorbidities (including osteoarthritis of the hip or knee, chronic lung disease, cardiac disease, and neurologic diseases) and body mass index (BMI, continuous variable), psychological health (anxiety (HADS anxiety score $\geq 8$ ), depression (HADS depression score $\geq 8$ ), and fear avoidance beliefs (FABQ-P $>16$ ), and current episode (duration of complaints $<3$ months, 3-6 months, 6-12 months, $>12$ months), baseline SSM symptoms, SSM function, and EQ-5D sum score.

To reduce the number of confounders included in the multiple regression model simultaneously, we used an approach based on change in the specific estimated effect of FABQ-P on each of the dichotomous outcomes. We considered those variables to be confounders that changed this effect by more than $\pm 10 \% .^{29}$

Some of the potential confounders had a small percentage of missing values (ranging from $0.4 \%$ to $4.7 \%$ ). We used multiple imputation based on chained equations (with five replications) to obtain data sets without missing values. ${ }^{30}$ The multiple imputation was based on the above described set of confounders, FABQ-P, and SSM symptoms and function. Pooled effect estimates were obtained with Rubin formula. ${ }^{31}$

All analyses were conducted with $\mathrm{R}$ for Windows. ${ }^{32}$

\section{Ethics}

This multicenter cohort study was conducted in compliance with all international laws and regulations as well as any applicable guidelines. The study was approved by the independent Ethics Committee of the Canton Zurich (KEK-ZHNR: 2010-0395/0).

\section{RESULTS}

\section{Patient Characteristics}

Between December 2010 and August 2015, 1537 patients were potentially eligible, and 800 patients agreed to participate (Figure 1, study flow). Decompression surgery was performed in 331 patients. For this study, 234 patients $(71 \%)$ completed the 12 months follow-up until the end of August 2015 and were included in the analysis.

In Table 1 we present the patients baseline characteristics for all patients and patients with low FABQ-P (194 patients with FABQ-P $\leq 16$ at baseline and/or at 6 months follow-up) and patients in group high FABQ-P-persistent (40 patients with FABQ-P $>16$ at baseline and 6 months follow-up).

\section{Continuous FABQ-P at Baseline and at 6 Months as Prognostic Indicator-Models Ia and Ib}

In the model Ia we found that the effect of cont. FABQ-P-0 was not linear on MCID in SSM symptoms and disability, respectively, at 12 months. Therefore, the linearity assumption was falsified and we restrained from further analysis.

In the model Ib, the effect of FABQ-P was linear on MCID in SSM symptoms and disability. The estimated OR for a one-unit change in FABQ-P-6 was 0.95 (95\% CI: $0.92-0.99)$ and 0.96 (95\% CI: 0.93-1.00) for MCID in SSM symptoms and disability, respectively, at 12 months.

\section{High FABQ-P-0 (at Baseline) as Prognostic Indicator-Model Ila}

We found in the simple logistic regression analysis almost no effect of high FABQ-P-0 for MCID in SSM symptoms at 12 months (OR 0.95; 95\% CI: $0.55-1.67)$ and for MCID in SSM function at 12 months (OR 1.11; 95\% CI: 0.64-1.92). The minimal effect of FABQ-P-0 on all outcomes might have resulted in an overestimated effect of confounders. Therefore, we restrained from further confounder analysis.

\section{High FABQ-P-6 (at 6 Months) as Prognostic Indicator-Model IIb}

In the model IIb we found in the simple logistic regression analysis an effect of high FABQ-P-6 for MCID in SSM symptoms at 12 months (OR 0.58; 95\% CI: 0.31-1.09). In the high FABQ-P-6 group patients were less likely to report a MCID than patients in the low FABQ-P-6 group $(58.9 \%$ vs. $72.5 \%)$. In the final multiple logistic regression 


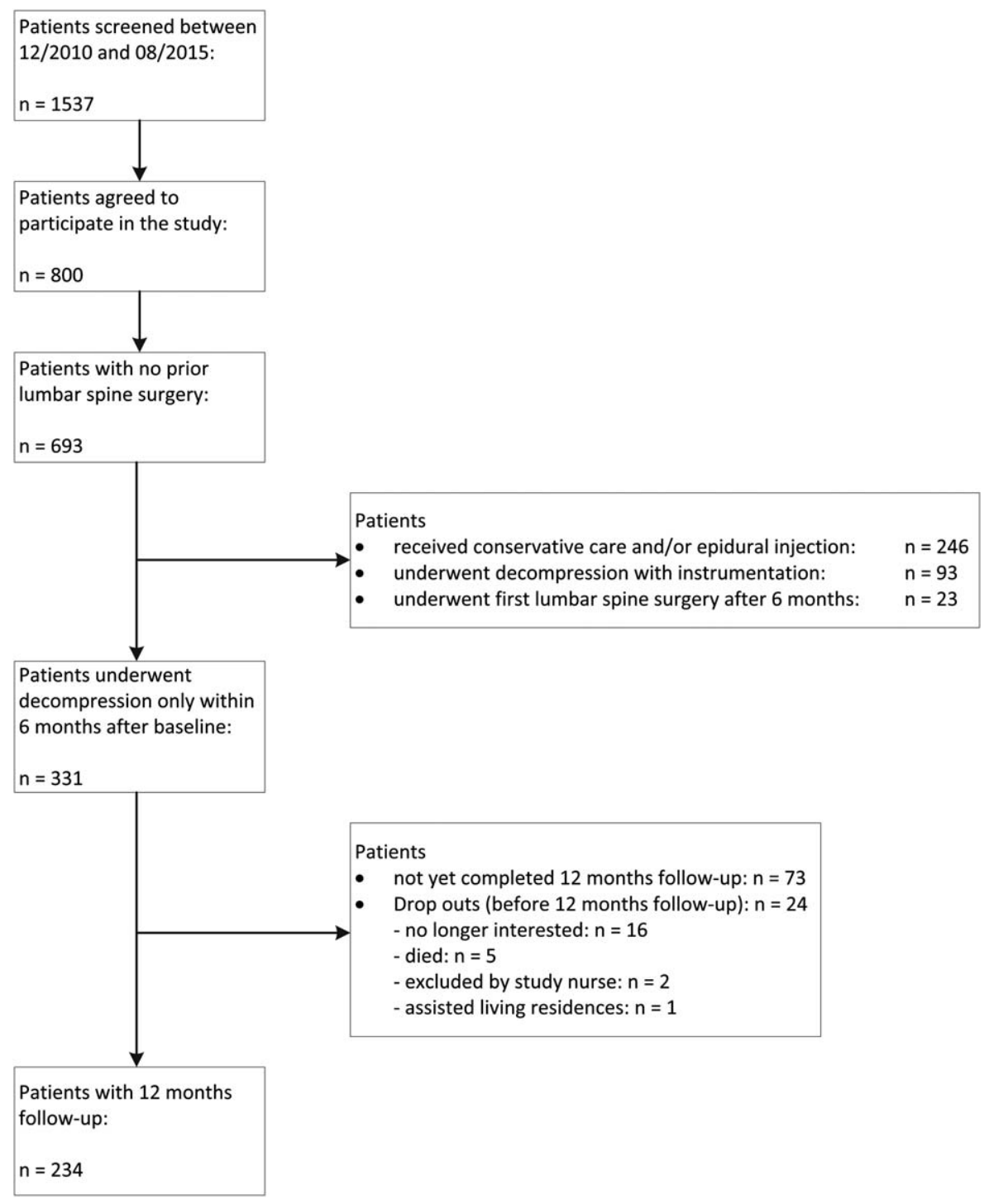

Figure 1. Study flow.

model we found that patients with high FABQ-P-6 were less likely to report MCID in SSM symptoms scale at 12 months (OR 0.46; 95\% CI: 0.24-0.91) after adjustment for confounding (Table 2).

For disability, the simple logistic regression analysis showed an effect of high FABQ-P-6 for MCID in SSM function at 12 months (OR 0.82; 95\% CI: 0.43-1.55). Patients in the high FAB-P-6 group reported similar MCID rates compared to the low FABQ-P-6 group $(64.3 \% v s$. $68.9 \%$ ). In the final multiple logistic regression model patients with high FABQ-P-6 showed a trend toward less MCID in SSM function at 12 months (OR 0.64; 95\% CI: 0.31-1.32) after adjustment for confounding, however, the results were not statistically significant (Table 2).

\section{High FABQ-P-Persistent (at Baseline and 6 Months) as Prognostic Indicator-Model III}

In the model III we found in the simple logistic regression analysis an effect of high FABQ-P-persistent for MCID in SSM symptoms at 12 months (OR 0.49; 95\% CI: 0.24-0.98). The percentage of patients who reached MCID was smaller than in the low FABQ-P-persistent group $(55 \%$ and $72.3 \%$, respectively). In the multiple logistic regression model patients with high FABQ-P-persistent were less likely to report MCID in SSM symptoms at 12 months (OR 0.34, 95\% CI: 0.16-0.73) after adjustment for confounding (Table 3 ).

For disability, the effect of high FABQ-P-persistent showed in the simple logistic regression analysis an effect for MCID in SSM function at 12 months (OR 0.69; 95\% CI: 0.34-1.4). The percentage of patients who reached MCID was similar between the high and low FABQ-P-persistent groups $(60 \%$ and $68.6 \%$, respectively). In the multiple logistic regression model patients with high FABQ-P-persistent were again less likely to experience MCID at 12 months (OR 0.53, 95\% CI: 0.25-1.15) after adjustment for confounding. While the effect was relevant, the wide $95 \%$ CI crossed one and therefore, the effect was not statistically significant (Table 3). 


\begin{tabular}{|c|c|c|c|c|}
\hline & All & Low FABQ-P* & $\begin{array}{c}\text { High FABQ-P- } \\
\text { Persistent }^{\dagger}\end{array}$ & $P$ Value \\
\hline $\mathrm{N}$ & 234 & 194 & 40 & \\
\hline Age, years, median [IQR] & $75.0[68,80]$ & $75.0[68,80]$ & $74.5[68,81]$ & 0.9 \\
\hline Female, n (\%) & $120(51.3)$ & $99(51)$ & $21(52.5)$ & 0.99 \\
\hline $\mathrm{BMI}, \mathrm{kg} / \mathrm{m}^{2}$, median $[\mathrm{IQR}]$ & $26.9[24.3,30.4]$ & $26.8[24.4,30.3]$ & $27.4[23.8,30.5]$ & 0.97 \\
\hline Diabetes, $\mathrm{n}(\%)$ & $34(14.5)$ & $26(13.4)$ & $8(20)$ & 0.41 \\
\hline Education, $\mathrm{n}(\%)$ & & & & 0.08 \\
\hline Compulsory school & $58(24.8)$ & $53(27.3)$ & $5(12.5)$ & \\
\hline $\begin{array}{l}\text { High school, college, } \\
\text { university }\end{array}$ & $176(75.2)$ & $141(72.7)$ & $35(87.5)$ & \\
\hline Civil status, $\mathrm{n}(\%)$ & & & & 0.88 \\
\hline Single, divorced, widowed & $94(40.2)$ & 77 (39.7) & $17(42.5)$ & \\
\hline $\begin{array}{l}\text { Married, registered } \\
\text { partnership }\end{array}$ & $140(59.8)$ & $117(60.3)$ & $23(57.5)$ & \\
\hline Duration of complaints, $\mathrm{n}(\%)$ & & & & 0.58 \\
\hline$<3$ months & $25(10.8)$ & $19(9.9)$ & $6(15.4)$ & \\
\hline $3-6$ months & $42(18.2)$ & $36(18.8)$ & $6(15.4)$ & \\
\hline $6-12$ months & $32(13.9)$ & $25(13.0)$ & $7(17.9)$ & \\
\hline$>12$ months & $132(57.1)$ & $112(58.3)$ & $20(51.3)$ & \\
\hline \multicolumn{5}{|l|}{ Comorbidities, n (\%) } \\
\hline Coxarthrosis & $32(14.0)$ & $25(13.2)$ & $7(17.9)$ & 0.6 \\
\hline Gonarthrosis & $37(16.2)$ & $30(15.9)$ & $7(17.9)$ & 0.93 \\
\hline $\begin{array}{l}\text { Chronic lung disease } \\
\text { (COPD, asthma, fibrosis, } \\
\text { others) }\end{array}$ & $13(5.7)$ & $7(3.7)$ & $6(15)$ & 0.02 \\
\hline Heart failure & $14(6.1)$ & $10(5.3)$ & $4(10)$ & 0.44 \\
\hline Coronary disease & $17(7.4)$ & $11(5.8)$ & $6(15)$ & 0.09 \\
\hline Neuropathy & $21(9.2)$ & $15(7.9)$ & $6(15.4)$ & 0.25 \\
\hline Parkinson disease & $5(2.2)$ & $5(2.6)$ & $0(0)$ & 0.66 \\
\hline SSM symptoms, median [IQR] & $3.1[2.7,3.6]$ & $3.1[2.7,3.4]$ & $3.4[3.0,3.7]$ & 0.01 \\
\hline SSM function, median [IQR] & $2.4[1.8,2.8]$ & $2.3[1.8,2.8]$ & $2.7[2,3]$ & 0.05 \\
\hline $\begin{array}{l}\text { EQ5D sum score, median } \\
\text { [IQR] }\end{array}$ & $70[60,80]$ & $70[60,80]$ & $60[50,80]$ & 0.08 \\
\hline HADS anxiety, n (\%) & & & & 0.44 \\
\hline $0-7$ & $194(82.9)$ & $163(84)$ & $31(77.5)$ & \\
\hline $8-21$ & $40(17.1)$ & $31(16)$ & $9(22.5)$ & \\
\hline HADS depression, $\mathrm{n}(\%)$ & & & & 0.3 \\
\hline $0-7$ & $192(82)$ & $162(83.4)$ & $30(75)$ & \\
\hline $8-21$ & $42(18)$ & $32(16.6)$ & $10(25)$ & \\
\hline $\begin{array}{l}\text { FABQ-P-0 (at baseline), } \\
\text { median [IQR] }\end{array}$ & $16[12,20]$ & $15[10.8,18]$ & $22[19.8,24]$ & $<0.01$ \\
\hline $\begin{array}{l}\text { FABQ-P-6 (at } 6 \text { months), } \\
\text { median [IQR] }\end{array}$ & $12[4,16.5]$ & $8[1.5,14]$ & $21[19,23]$ & $<0.01$ \\
\hline \multicolumn{5}{|c|}{$\begin{array}{l}{ }^{*} F A B Q-P \leq 16 \text { at baseline and/or } \leq 16 \text { at } 6 \text { months. } \\
{ }^{\top} F A B Q-P>16 \text { at baseline and at } 6 \text { months. } \\
\text { BMI indicates body mass index; FABQ-P, Fear Avoidance Beliefs Physical Activity Subscale; HADS, Hospital Anxiety and Depression Scale; SSM, spina } \\
\text { stenosis measure. }\end{array}$} \\
\hline
\end{tabular}

\section{DISCUSSION}

In this analysis of 234 patients undergoing decompression surgery for symptomatic degenerative LSS we found baseline high FAB measured by FABQ-P not to be associated with pain of symptoms and disability at 12 months' follow-up. For continuous FABQ-P-0 we found no linear association and for
FABQ-P-6 there was an effect of about 5\% reduced chance for MCID on SSM symptoms and function per one unit change at 12 months. Patients with high FABQ-P-6 and high FABQ-P-persistent were less likely to report a MCID for SSM symptoms at 12 months. Our analysis found a similar trend for disability. However, the confidence intervals were wide 
depression, and maintains the original negative appraisal in a deleterious cycle. In low back pain high FAB were indicators for poor outcome in the subacute phase and influenced the treatment response in conservative treatments. ${ }^{12,13}$ In patients undergoing surgery for spinal disorders one study showed a postoperative reduction in fear avoidance beliefs. ${ }^{8}$ Similar to our study, they found that preoperative fear of movement (measured by the Tampa Scale of kinesiophobia) was not a prognostic factor for poor outcome. Archer et $a l^{8}$ found in their study that postoperative high scores in the Tampa Scale at 6 weeks and 3 months were associated with more pain and disability.

Wood et $a l^{33}$ compared the influence of fear of movement (measured by the Tampa Scale of kinesiophobia) in patients with lumbar spinal stenosis with neurogenic claudication to patients with claudication due to peripheral arterial disease and asymptomatic patients. In this cross-sectional study they found that persons with neurogenic claudication reported higher fear avoidance beliefs than patients with claudication due to other illnesses.

A study including 159 patients with lumbar spinal stenosis or disc herniation that underwent decompression surgery showed in a prediction model that preoperative $\mathrm{FAB}$ of physical activity was the only predictor for postoperative outcome (measured by using a global outcome measure) after 1 year. ${ }^{34}$ In the study by Havakeshian and Mannion, ${ }^{34}$ FABQ-P were used on a continuous scale. Our study aimed to investigate the impact of high FABQ-P beliefs using a cutoff and therefore, increasing the clinical applicability. In our study in elderly patients with symptomatic degenerative lumbar spinal stenosis undergoing decompressive surgery high preoperative FABQ-P were not a prognostic indicator for MCID at 6 or 12 months for patient reported outcomes pain and disability. High FABQ-P-6 and high FABQ-Ppersistent were associated with less MCID for pain at 12 months but not for disability. One explanation could be that the FABQ-P was originally validated for low back pain patients. The wording focuses on pain and the back. In LSS patients the complaints are frequently not primarily back related or the pain itself but rather the neurogenic complaints or gait impairment. Hence, we found an association between postoperative FABQ-P and pain outcomes but not on disability. Therefore, the wording of the FABQ-P may need to be adjusted and the scale validated for this specific population. One other explanation could be that preoperative fear (fear of the pending surgery) might influence the reporting in FABQ-P. The FABQ-P does not specifically assess surgical fear and therefore, we were not able to distinguish between surgical fear and FABQ-P as negative coping strategy. ${ }^{6}$

Preoperative fear has been shown in patients undergoing elective surgery to be associated with acute postoperative as well as long-term pain. ${ }^{35}$ Our findings indicated that high FABQ-P-6 or high FABQ-P-persistent were a relevant factor for pain at 12 months but not disability. It can be hypothesized that in patients with high FABQ-P-persistent fear persists over a longer time period and so the impact will be more relevant on their ability to cope with the pain. One may also hypothesize that high fear avoidance beliefs are the result and not the cause of a worse treatment outcome. The correlations between FABQ-P-0 with symptoms and disability at baseline and the FABQ-P-6 with symptoms and disability at 6 months, respectively, were weak (data not shown). This indicates that high FABQ-P-persistent after surgery is independent of symptoms and disability a relevant prognostic indicator that may be modified by specific treatment approaches. This has to be addressed specifically in future studies.

\section{Strength and Limitations}

A limitation of this study was that it was not possible to distinguish how strongly FABQ-P-0 was influenced by specifically assessed surgical fear. Furthermore, our cutoff value for FABQ-P was derived based on the 75 th percentile according to a previously published study; however, a variety of other cutoff values have been proposed but none of them has been established yet. Additionally, we did not evaluate catastrophizing, and thus we were unable to assess the influence of catastrophizing on the interaction between FABQ-P and catastrophizing.

Our study has several strengths. These include the multicenter setting and prospective collection of data, as well as the use of established questionnaires on degenerative lumbar spinal stenosis. Furthermore, we had a homogenous patient sample regarding the treatment since only first-time decompression surgeries without fusions were included. Additionally, we used multiple imputation techniques to avoid potentially biased results as compared with a complete case analysis.

\section{Implications for Research}

Our finding was unexpected considering that high preoperative $\mathrm{FAB}$ has been shown in a previous study to be a predictor of poor outcome. ${ }^{34}$ Future studies should further investigate the importance of fear of surgery and its difference to $\mathrm{FAB}$ as a negative coping. In future studies on patients undergoing surgical treatment it is advisable to use additionally a specific questionnaire assessing surgical fear, such as the Surgical Fear Questionnaire (SFQ). ${ }^{35}$ Furthermore, it is unclear whether patients with persistent fear avoidance beliefs after surgery may benefit from specific cognitive and behavioral intervention that have been shown in other setting to modify the impact of FAB.

\section{Implications for Clinical Practice}

In patients undergoing surgery for degenerative lumbar spinal stenosis high FABQ-P-0 were not associated with poor prognosis. Similar to previous studies we showed that FABQ-P-6 and FABQ-P-persistent may be important factors to consider in patients with persistent complaints.

\section{CONCLUSIONS}

In elderly patients undergoing decompression surgery for symptomatic degenerative lumbar spinal stenosis preoperative fear avoidance beliefs were not a prognostic indicator 
for the outcome. Patients with fear avoidance beliefs at 6 months and persistent fear avoidance beliefs were less likely to experience clinically relevant improvement in pain at 12 months. Studies should address the importance of persistent postoperative fear avoidance beliefs.

\section{Key Points}

To the present, the influence of pre- and postoperative $F A B$ on the prognosis after surgery for LSS is still unclear.

$\square$ In the data of the LSOS preoperative FAB were not a prognostic indicator for the outcome pain or disability in patients undergoing decompression surgery for symptomatic degenerative LSS.

口 Patients with postoperative FAB were less likely to experience clinically relevant improvement in pain at 12 months.

- Studies should address the importance of persistent postoperative FAB.

\section{Acknowledgment}

The authors thank Dr. Sherri Weiser, psychologist at the Occupational and Industrial Orthopaedic Center (OIOC), New York University (NYU) Langone Medical Center, New York, for the very valuable discussions and her input.

\section{References}

1. Hoy D, March L, Brooks P, et al. The global burden of low back pain: estimates from the Global Burden of Disease 2010 study. Ann Rheum Dis 2014;73:968-74.

2. Deyo RA. Treatment of lumbar spinal stenosis: a balancing act. Spine J 2010;10:625-7.

3. Atlas SJ, Keller RB, Wu YA, et al. Long-term outcomes of surgical and nonsurgical management of lumbar spinal stenosis: 8 to 10 year results from the maine lumbar spine study. Spine (Phila Pa 1976) 2005;30:936-43.

4. Weinstein JN, Tosteson TD, Lurie JD, et al. Surgical versus nonoperative treatment for lumbar spinal stenosis four-year results of the Spine Patient Outcomes Research Trial. Spine (Phila Pa 1976) 2010;35:1329-38.

5. Brodner G, Mertes N, Buerkle H, et al. Acute pain management: analysis, implications and consequences after prospective experience with 6349 surgical patients. Eur J Anaesthesiol 2000;17:566-75.

6. Theunissen M, Peters ML, Bruce J, et al. Preoperative anxiety and catastrophizing: a systematic review and meta-analysis of the association with chronic postsurgical pain. Clin J Pain 2012;28: 819-41.

7. Sommer M, de Rijke JM, van Kleef M, et al. Predictors of acute postoperative pain after elective surgery. Clin J Pain 2010;26:87-94.

8. Archer KR, Wegener ST, Seebach C, et al. The effect of fear of movement beliefs on pain and disability after surgery for lumbar and cervical degenerative conditions. Spine (Phila Pa 1976) 2011;36:1554-62.

9. Linton SJ, Shaw WS. Impact of psychological factors in the experience of pain. Phys Ther 2011;91:700-11.

10. Wertli MM, Burgstaller JM, Weiser S, et al. Influence of catastrophizing on treatment outcome in patients with nonspecific low back pain: a systematic review. Spine (Phila Pa 1976) 2014;39:263-73.

11. Wertli MM, Eugster R, Held U, et al. Catastrophizing-a prognostic factor for outcome in patients with low back pain: a systematic review. Spine J 2014;14:2639-57.
12. Wertli MM, Rasmussen-Barr E, Held U, et al. Fear-avoidance beliefs-a moderator of treatment efficacy in patients with low back pain: a systematic review. Spine J 2014;14:2658-78.

13. Wertli MM, Rasmussen-Barr E, Weiser S, et al. The role of fear avoidance beliefs as a prognostic factor for outcome in patients with nonspecific low back pain: a systematic review. Spine J 2014;14:816-36.

14. Steurer J, Nydegger A, Held U, et al. LumbSten: the lumbar spinal stenosis outcome study. BMC Musculoskelet Disord 2010;11:254.

15. Waddell G, Newton M, Henderson I, et al. A Fear-Avoidance Beliefs Questionnaire (FABQ) and the role of fear-avoidance beliefs in chronic low back pain and disability. Pain 1993;52:157-68.

16. Swinkels-Meewisse EJ, Swinkels RA, Verbeek AL, et al. Psychometric properties of the Tampa Scale for kinesiophobia and the fear-avoidance beliefs questionnaire in acute low back pain. Man Ther 2003;8:29-36.

17. Stucki G, Liang MH, Fossel AH, et al. Relative responsiveness of condition-specific and generic health status measures in degenerative lumbar spinal stenosis. J Clin Epidemiol 1995;48:1369-78.

18. Tuli SK, Yerby SA, Katz JN. Methodological approaches to developing criteria for improvement in lumbar spinal stenosis surgery. Spine (Phila Pa 1976) 2006;31:1276-80.

19. Zucherman JF, Hsu KY, Hartjen CA, et al. A multicenter, prospective, randomized trial evaluating the $\mathrm{X}$ STOP interspinous process decompression system for the treatment of neurogenic intermittent claudication: two-year follow-up results. Spine (Phila Pa 1976) 2005;30:1351-8.

20. Hansraj KK, O’Leary PF, Cammisa FP Jr, et al. Decompression, fusion, and instrumentation surgery for complex lumbar spinal stenosis. Clin Orthop Relat Res 2001;18-25.

21. Fokter SK, Yerby SA. Patient-based outcomes for the operative treatment of degenerative lumbar spinal stenosis. Eur Spine J 2006;15:1661-9.

22. Hinz A, Klaiberg A, Brahler E, et al. The Quality of Life Questionnaire EQ-5D: modelling and norm values for the general population. Psychother Psychosom Med Psychol 2006;56:42-8.

23. Zigmond AS, Snaith RP. The hospital anxiety and depression scale. Acta Psychiatrica Scand 1983;67:361-70.

24. Crawford JR, Henry JD, Crombie C, et al. Normative data for the HADS from a large non-clinical sample. Br J Clin Psychol 2001;40:429-34.

25. Grotle M, Brox JI, Glornsrod B, et al. Prognostic factors in first-time care seekers due to acute low back pain. Eur J Pain 2007;11:290-8.

26. Beaton DE, Boers M, Wells GA. Many faces of the minimal clinically important difference (MCID): a literature review and directions for future research. Curr Opin Rheumatol 2002;14: 109-14.

27. Pincus T, Santos R, Breen A, et al. A review and proposal for a core set of factors for prospective cohorts in low back pain: a consensus statement. Arthritis Rheum 2008;59:14-24.

28. Deyo RA, Dworkin SF, Amtmann D, et al. Report of the NIH Task Force on research standards for chronic low back pain. Pain Med 2014;15:1249-67.

29. Rothman KJ, Greenland S, Lash TL. Introduction to Stratified Analysis. Modern Epidemiology. 3rd ed. Philadelphia, PA: Lippincott Williams \& Wilkins; 2008; 258-282.

30. van Buuren S, Groothuis-Oudshoorn K. mice: Multivariate Imputation by Chained Equations in R. J Stat Softw 2011;45:67.

31. Rubin DB. Multiple Imputation for Nonresponse in Surveysed. New York: John Wiley \& Sons; 2004.

32. R Core Team. R: A Language and Environment for Statistical Computing. R Foundation for Statistical Computing, Vienna, Austria. 2016

33. Wood D W, Haig A J, Yamakawa K S J. Fear of movement/ (re)injury and activity avoidance in persons with neurogenic versus vascular claudication. Spine J 2012;12:292-300.

34. Havakeshian S, Mannion AF. Negative beliefs and psychological disturbance in spine surgery patients: a cause or consequence of a poor treatment outcome?. Eur Spine J 2013;22:2827-35.

35. Theunissen M, Peters ML, Schouten EG, et al. Validation of the surgical fear questionnaire in adult patients waiting for elective surgery. PLoS One 2014;9:e100225. 